University of Nebraska - Lincoln

DigitalCommons@University of Nebraska - Lincoln

Faculty Publications from the Department of Electrical \& Computer Engineering, Department Electrical and Computer Engineering

2010

\title{
Magneto-optical properties of cobalt slanted columnar thin films
}

\author{
Daniel Schmidt \\ Tino Hofmann \\ thofmann4@unl.edu \\ Craig M. Herzinger \\ Eva Schubert \\ efranke3@unl.edu \\ Mathias Schubert \\ mschubert4@unl.edu
}

Follow this and additional works at: https://digitalcommons.unl.edu/electricalengineeringfacpub

Part of the Electrical and Computer Engineering Commons

Schmidt, Daniel; Hofmann, Tino; Herzinger, Craig M.; Schubert, Eva; and Schubert, Mathias, "Magnetooptical properties of cobalt slanted columnar thin films" (2010). Faculty Publications from the Department of Electrical and Computer Engineering. 111.

https://digitalcommons.unl.edu/electricalengineeringfacpub/111

This Article is brought to you for free and open access by the Electrical \& Computer Engineering, Department of at DigitalCommons@University of Nebraska - Lincoln. It has been accepted for inclusion in Faculty Publications from the Department of Electrical and Computer Engineering by an authorized administrator of DigitalCommons@University of Nebraska - Lincoln. 


\title{
Magneto-optical properties of cobalt slanted columnar thin films
}

\author{
Daniel Schmidt, ${ }^{1, a)}$ Tino Hofmann, ${ }^{1}$ Craig M. Herzinger, ${ }^{2}$ Eva Schubert, ${ }^{1}$ and \\ Mathias Schubert ${ }^{1}$ \\ ${ }^{1}$ Department of Electrical Engineering and Nebraska Center for Materials and Nanoscience, \\ University of Nebraska-Lincoln, Lincoln, Nebraska 68588-0511, USA \\ ${ }^{2}$ J. A. Woollam Co. Inc., 645 M Street, Suite 102 Lincoln, Nebraska 68508-2243, USA
}

(Received 3 December 2009; accepted 9 February 2010; published online 2 March 2010)

\begin{abstract}
Magneto-optical properties of ferromagnetic slanted columnar thin films from cobalt made by electron-beam glancing angle deposition are reported. Magneto-optic ellipsometry in the polar Kerr effect configuration was employed to determine the field- and wavelength-dependent complex magneto-optic polarizability parameter within the spectral range from 0.74 to $3.0 \mathrm{eV}$. Kerr effect measurements and calculations reveal a strong azimuthal dependence for the incident linear polarization with peak Kerr rotation one order of magnitude larger than what has been reported for solid Co thin films. () 2010 American Institute of Physics. [doi:10.1063/1.3340913]
\end{abstract}

With the introduction of advanced growth techniques such as glancing angle deposition, engineered threedimensional metamaterials on the nanometer scale are available. ${ }^{1,2}$ Sculptured thin films can be tailored from many materials to achieve desired physical properties that can vary significantly from bulk properties due to size and confinement effects. Ferromagnetic materials such as cobalt are particularly interesting for future magneto-optical applications since magnetic and optical properties may be tailored at the same time. Nanostructured ferromagnets may be used for thin film based magneto-optical devices such as optical diodes or Faraday rotation cells. Slanted columnar thin films (SCTFs) comprise rather simple geometries yet show complex anisotropic optical, magnetic, and magneto-optical properties. ${ }^{3-5}$ Due to a relatively low extinction coefficient, high penetration depths can be achieved in SCTFs for electromagnetic radiation at visible wavelengths. Therefore, the magnetic field-induced birefringence is no longer probed only at the near surface, as with highly absorbing metal films. For example, such ferromagnetic nanoscaffolds may be interesting for low-loss transmission magneto-optical modulators. We have recently reported on the determination of principal dielectric functions of birefringent SCTFs from cobalt. ${ }^{3}$ In this letter, we focus on the determination of the wavelength-dependent complex magneto-optic polarizability parameter $\varepsilon_{x y}^{\mathrm{MO}}$ of cobalt SCTFs and the resulting anisotropic Kerr rotation and ellipticity. Magneto-optic materials can be characterized by means of generalized magneto-optic ellipsometry. ${ }^{6,7}$ Generalized ellipsometry (GE), a nondestructive and noninvasive optical technique, has proven to be highly suitable for determining optical properties of highly anisotropic structured films from metals such as SCTFs or helical sculptured thin films. ${ }^{3,8,9}$ Measurement of the complex ratio $\rho$ of the $s$ - and $p$-polarized reflectivities is presented here in terms of the Stokes descriptive system, where real-valued Mueller matrix elements $\mathbf{M}_{i j}$ connect the Stokes parameters before and after sample interaction. ${ }^{10}$ The linear polarizability response of structured thin films due to an electric field $\mathbf{E}$ is a superposition of contributions along certain directions $\mathbf{a}=a_{x} \mathbf{x}+a_{y} \mathbf{y}+a_{z} \mathbf{z}, \quad \mathbf{b}=b_{x} \mathbf{x}+b_{y} \mathbf{y}+b_{z} \mathbf{z}, \quad \mathbf{c}=c_{x} \mathbf{x}$

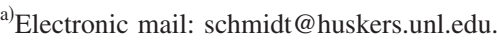

$+c_{y} \mathbf{y}+c_{z} \mathbf{z} ; \mathbf{P}=\varrho_{a} \mathbf{a}+\varrho_{\mathbf{b}} \mathbf{b}+\varrho_{\mathbf{c}} \mathbf{c}$, that are phenomenologically equivalent to (but physically different from) the crystallographic unit-cell vectors in bulk materials. ${ }^{11}$ In the laboratory Cartesian coordinate system the SCTF response is described by the second rank susceptibility tensor $\chi$ and $\mathbf{P}=(\mathbf{1}-\boldsymbol{\varepsilon}) \mathbf{E}$ $=\chi \mathbf{E}$. Axes $\mathbf{a}, \mathbf{b}$, and $\mathbf{c}$ span a monoclinic coordinate system with $\mathbf{c}$ along the long axis of the columns, a perpendicular to $\mathbf{b}$ and $\mathbf{c}$ and parallel to the substrate surface, and a monoclinic angle $\beta$ between $\mathbf{c}$ and $\mathbf{b} .{ }^{8,9}$ Ellipsometric data analysis for anisotropic thin film samples requires nonlinear regression methods, where measured and calculated GE data are matched as close as possible by varying appropriate physical model parameters. Due to the complexity of this subject, thorough discussion of this issue is beyond the scope of this paper, and referral is made to the literature. ${ }^{10,12}$ As a result of data analysis, we obtain major-axes polarization response functions $\varrho_{a}, \varrho_{b}, \varrho_{c}$, Euler angles $\varphi, \psi, \theta$, internal angles $\alpha, \beta, \gamma$, and thin film thickness $d .{ }^{11}$ The major optical constants $\left(n_{j}+i k_{j}\right) \equiv \sqrt{\left(1+\varrho_{j}\right)}(j=a, b, c)$ can be extracted on a wavelength-by-wavelength basis, i.e., without physical line shape implementations. Kramers-Kronig consistency tests can then be done individually for dielectric functions along each axis. ${ }^{11}$ However, a common, generally more robust procedure is matching parameterized model dielectric functions (MDFs) to experimental data simultaneously for all spectral data points. Parametric models further prevent wavelengthby-wavelength measurement noise from becoming part of the extracted dielectric functions and greatly reduce the number of free parameters.

In the presence of an external magnetic field, the dielectric tensor has to be augmented by off-diagonal complex parameters, which account for magneto-optical activity. ${ }^{13}$ In the polar Kerr effect configuration, with magnetic field orientation parallel to the surface normal (z-axis), the complex dielectric tensor of a monoclinic dielectric and uniaxial magneto-optic sample takes the following form:

$$
\begin{aligned}
& \varepsilon=\mathbf{A}(\varphi, \theta) \varepsilon^{\mathrm{D}} \mathbf{A}^{-1}(\varphi, \theta)+\varepsilon^{\mathrm{MO}}, \\
& \varepsilon^{\mathrm{D}}=\left(\begin{array}{ccc}
1+\varrho_{a} & 0 & 0 \\
0 & 1+\sin ^{2} \beta \varrho_{b} & \sin \beta \cos \beta \varrho_{b} \\
0 & \sin \beta \cos \beta \varrho_{b} & 1+\cos ^{2} \beta \varrho_{b}+\varrho_{c}
\end{array}\right),
\end{aligned}
$$




$$
\varepsilon^{\mathrm{MO}}=\left(\begin{array}{ccc}
0 & i \varepsilon_{x y}^{\mathrm{MO}} & 0 \\
-i \varepsilon_{x y}^{\mathrm{MO}} & 0 & 0 \\
0 & 0 & 0
\end{array}\right),
$$

where $\mathbf{A}(\varphi, \theta)$ is the usual Euler angle rotation matrix, ${ }^{12}$ which accounts for slanting angle of the columns relative to the $z$-axis $(\theta)$ as well as in-plane orientation of the slanted columns $\varphi$ relative to the plane of incidence, $\varepsilon^{\mathrm{D}}$ represents the dielectric polarizability of a birefringent SCTF with monoclinic angle $\beta$, and $\varepsilon^{\mathrm{MO}}$ accounts for the magneto-optic polarizability. As discussed below, we find magneto-optic cylindrical symmetry around the $z$-axis, and $\varepsilon^{\mathrm{MO}}$ commutes with the rotation operation and can be separated in Eq. (1) without magneto-optical in-plane anisotropy. It should be pointed out that the polar Kerr effect is typically probed near normal incidence; hence the magneto-optical response is only due to an incoming electric field in-plane (parallel to the film interface).

Subsequently, Kerr rotation $\theta_{\mathrm{K}}$ and ellipticity $\varepsilon_{\mathrm{K}}$ can be calculated, which are in our notation identical to the magnetic field-induced changes in the normalized complex offdiagonal Jones matrix element $R_{p s}=r_{p s} / r_{p p}$ at normal incidence (i.e., $\left.\theta_{\mathrm{K}}+i \varepsilon_{\mathrm{K}}=R_{p s}\left[\mu_{0} H\right]-R_{p s}\left[\mu_{0} H=0 \mathrm{~T}\right]\right)^{7}$. Because $R_{p s}\left[\mu_{0} H=0 \mathrm{~T}\right]$ is not zero in general for anisotropic samples, the Kerr effect depends on the anisotropic optical properties of the sample and thus is a function of the incident linear polarization relative to the sample orientation.

Cobalt nanostructures and a $60 \mathrm{~nm}$ thick reference film were deposited at room temperature by electron-beam glancing angle deposition in a customized ultrahigh vacuum chamber. Details about the growth process and a representative image of a Co SCTF can be found elsewhere. ${ }^{3}$ Magnetooptic spectroscopic Mueller matrix measurements were performed in the polar Kerr effect setup near normal incidence $\left(\Phi_{\mathrm{a}}=3^{\circ}\right)$ within the spectral range from 0.74 to $3.0 \mathrm{eV}$ with a rotating compensator ellipsometer (V-VASE, J. A. Woollam Co., Inc.) at varying magnetic fields of $-1.8,0$, and $1.8 \mathrm{~T}$. The magnetic field was generated by a water-cooled splitcoil electromagnet. The reflected beam was guided by a mirror into the detector. ${ }^{14}$

In order to minimize cross-correlation between varying parameters sample analysis was done in three steps. First, multiple angle of incidence Mueller matrix ellipsometry measurements were carried out on an automated stage at different in-plane orientations $\varphi$ to accurately determine the anisotropic dielectric response function for Co SCTFs as reported previously. ${ }^{3}$ Other parameters determined from this investigation are total film thickness $d=107.9 \mathrm{~nm}$, slanting angle $\theta=63.3^{\circ}$, and monoclinic angle $\beta=81.0^{\circ}$. Subsequently, the sample was transferred to the polar magnetooptic Kerr effect setup and spectroscopic Mueller matrix measurements at $\mu_{0} H=0 \mathrm{~T}$ were analyzed using the model obtained in the first step, in order to determine sample azimuth and angle of incidence parameters. Measurements were then taken with applied external magnetic fields and only the wavelength-dependent complex magneto-optic polarizability parameter $\varepsilon_{x y}^{\mathrm{MO}}$ was determined by matching the calculated Mueller matrix difference $\Delta \mathrm{M}_{i j}=\mathrm{M}_{i j}\left(-\mu_{0} H\right)-\mathrm{M}_{i j}\left(\mu_{0} H\right)$ with the experiment. Data analysis of this final step was done both on a wavelength-by-wavelength basis as well as with a parameterized MDF. While data at different in-plane orientations $\varphi$ differ due to the monoclinic anisotropy, measure-

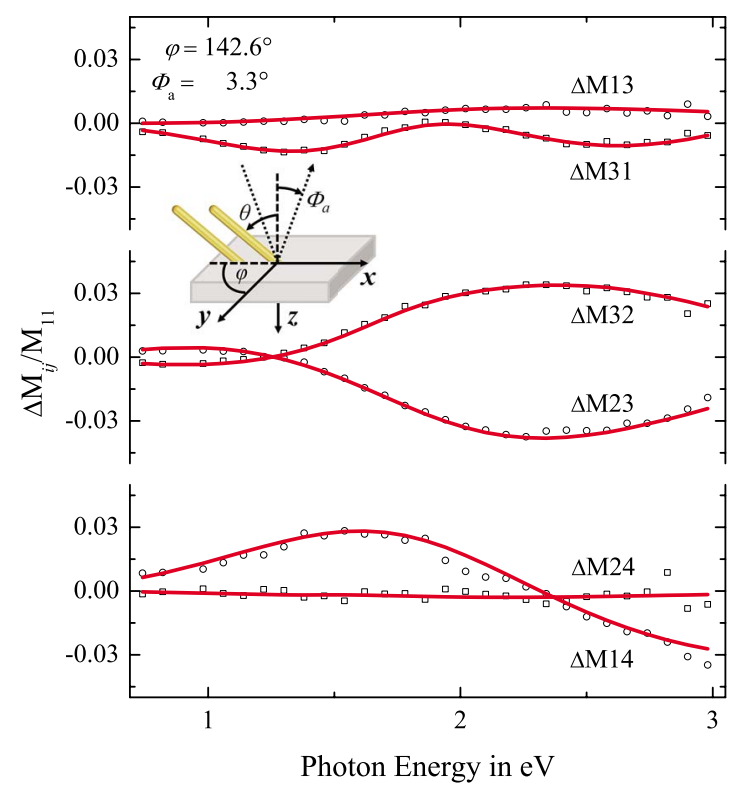

FIG. 1. (Color online) Experimental (symbols) and best-match model calculated (solid line) magnetic field-induced difference data $\Delta \mathrm{M}_{i j}=\mathrm{M}_{i j}\left(\mu_{0} H\right.$ $=-1.8 \mathrm{~T})-\mathrm{M}_{i j}\left(\mu_{0} H=1.8 \mathrm{~T}\right)$ normalized to $\mathrm{M}_{11}$ for a Co SCTF. The inset shows a schematic drawing of the sample with in-plane orientation $\varphi$, column tilt $\theta$, and angle of incidence $\Phi_{\mathrm{a}}$.

ments at multiple in-plane orientations, $\varphi=0^{\circ}, \pm 45^{\circ}$, and $180^{\circ}$, revealed no magneto-optic in-plane anisotropy. Therefore, we conclude that magnetic domains orient along the external magnetic field regardless of $\varphi$ (i.e., not along the columns) and the corresponding model scenario is represented by Eq. (1), where $\varepsilon^{\mathrm{MO}}$ possesses no $\varphi$ dependence.

Figure 1 shows experimental data as well as generated MDF data of selected elements of the Mueller matrix difference $\Delta \mathrm{M}_{i j}$ normalized to $\mathrm{M}_{11}$ for our Co SCTF at an external magnetic field $\mu_{0} H= \pm 1.8 \mathrm{~T}$ and in-plane orientation of $\varphi$ $=142.6^{\circ}$. Note that an in-plane orientation of $\varphi=90^{\circ}$ represents the situation where the slanted columns are parallel to the plane of incidence and pointing toward the source (see inset). Generated and experimental data are in excellent agreement. Note that data from multiple sample azimuth orientations were simultaneously included in the best-match model fitting procedure.

Real and imaginary parts of the complex magneto-optic polarizability function $\varepsilon_{x y}^{\mathrm{MO}}$ at $\mu_{0} H=1.8 \mathrm{~T}$ are depicted in Fig. 2, also in comparison with data obtained from the Co reference sample at $\mu_{0} H=1.5 \mathrm{~T}$, which are in agreement with existing literature. ${ }^{15}$ Symbols and solid lines represent results from a wavelength-by-wavelength and parameterized MDF analysis, respectively. The Kramers-Kronig consistent MDF for $\varepsilon_{x y}^{\mathrm{MO}}$ consists of two Lorentz oscillators centered at photon energies 0.84 and $1.64 \mathrm{eV}$, which account for the difference in left and right circularly polarized light spin-split transitions. ${ }^{16}$ Note that $\varepsilon_{x y}^{\mathrm{MO}}$ differs substantially between SCTF and Co thin solid film.

Figure 3 depicts calculated Kerr rotation $\theta_{\mathrm{K}}$ and Kerr ellipticity $\varepsilon_{\mathrm{K}}$ using functions $\varepsilon_{x y}^{\mathrm{MO}}$ (MDF) and experimental data at $\mu_{0} H=1.8 \mathrm{~T}$. The calculated spectra are shown for two different SCTF thickness values (left and right panel, respectively), as well as for two in-plane orientations, where the incident linear polarization is perpendicular to the columns at $\varphi=0$. Peak Kerr rotation values obtained for the Co SCTF are one order of magnitude larger than those reported 


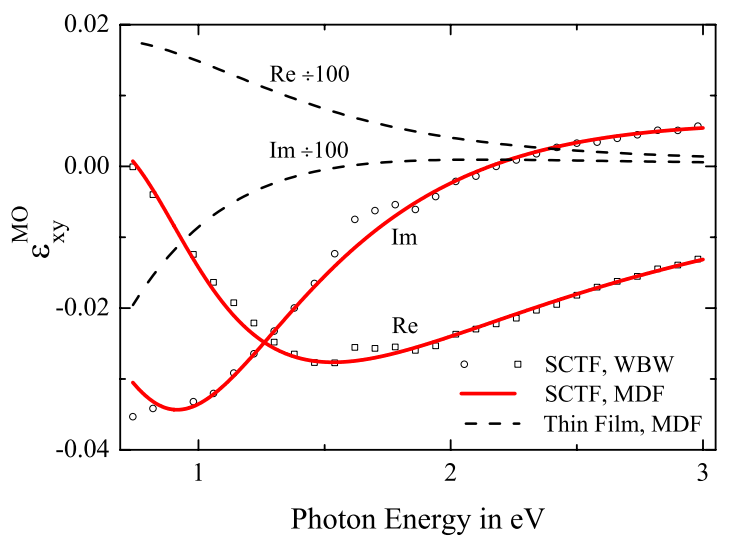

FIG. 2. (Color online) Real (Re) and imaginary (Im) parts of functions $\varepsilon_{x y}^{\mathrm{MO}}$ from a Co SCTF $\left(\mu_{0} H=1.8 \mathrm{~T}\right)$ and a $60 \mathrm{~nm}$ thick solid Co film $\left(\mu_{0} H\right.$ $=1.5 \mathrm{~T}$; spectra divided by 100). Symbols and solid lines for the SCTF are results from a wavelength-by-wavelength (WBW) and a model dielectric function (MDF) analysis, respectively, and experimental data shown in Fig. 1.

for solid Co thin films. ${ }^{17}$ These peaks in $\theta_{\mathrm{K}}$ and $\varepsilon_{\mathrm{K}}$ are caused by spectral minima in the $p-p$ polarized reflectance coefficients, $r_{p p}$, due to anisotropic interference within the SCTF. The anisotropic mode coupling described by the symmetric part of $\varepsilon$ in Eq. (1) enhances the Kerr effect generated by $\varepsilon^{\mathrm{MO}}$, and results in well measurable rotation and ellipticity signals. In order to achieve comparable large Kerr rotation values, complex rare-earth metal containing multilayer thin films have been necessary previously. ${ }^{18}$ Notably, an azimuthal rotation of $10^{\circ}$ causes $\theta_{\mathrm{K}}$ to alter orientation and has almost no influence on $\varepsilon_{\mathrm{K}}$. Maxima in $\theta_{\mathrm{K}}$ and $\varepsilon_{\mathrm{K}}$ coincide spectrally for a given thickness and can be shifted conve-

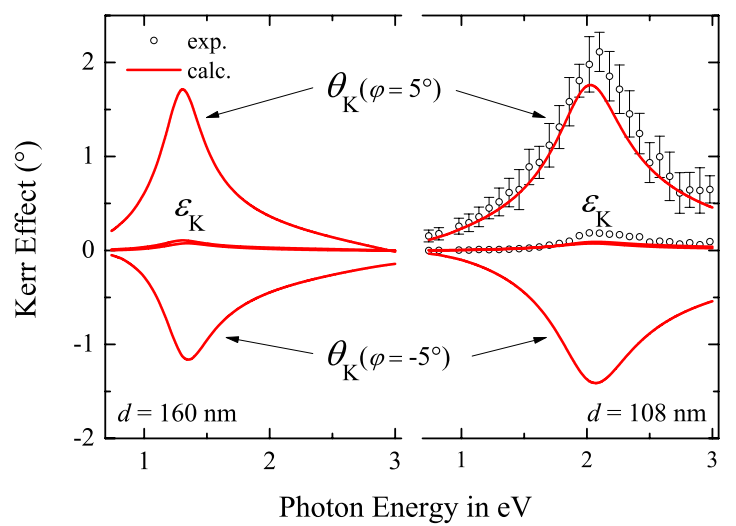

FIG. 3. (Color online) Experimental (symbols) and calculated (lines) data of Kerr rotation $\theta_{\mathrm{K}}$ and Kerr ellipticity $\varepsilon_{\mathrm{K}}$ at $\mu_{0} H=1.8 \mathrm{~T}$ of a Co SCTF at two different in-plane orientations $\varphi$ and thicknesses $d$. niently by varying the film thickness over the entire spectral range investigated here. Interestingly, due to the monoclinic anisotropy, sample azimuth variations with respect to the incident linear polarization have similar effects on Kerr parameters as a change in the external magnetic field direction.

In summary, we have determined the magneto-optical response of SCTF from cobalt and calculated the azimuthally dependent Kerr effect. The observed cylindrical symmetry of $\varepsilon_{x y}^{\mathrm{MO}}$ around the $z$-axis may be due to a polycrystalline nature of the Co SCTF. In contrast, due to intrinsic monoclinic anisotropy a highly anisotropic Kerr effect is predicted and observed by variations of the in-plane orientation, and which produces large Kerr rotation angles. Magneto-optical properties of SCTF can be tailored by choice of geometry and orientation.

The authors acknowledge financial support from the National Science Foundation in CAREER (Grant No. ECCS0846329), and MRSEC (Grant No. DMR-0820521), University of Nebraska-Lincoln, and J. A. Woollam Foundation.

${ }^{1}$ K. Robbie and M. J. Brett, J. Vac. Sci. Technol. A 15, 1460 (1997).

${ }^{2}$ M. M. Hawkeye and M. J. Brett, J. Vac. Sci. Technol. A 25, 1317 (2007).

${ }^{3}$ D. Schmidt, A. C. Kjerstad, T. Hofmann, R. Skomski, E. Schubert, and M. Schubert, J. Appl. Phys. 105, 113508 (2009). Note the misprint in Fig. 1, where axes $\mathbf{a}$ and $\mathbf{b}$ need to be exchanged. Same for Fig. 1 in Refs. 8 and 9.

${ }^{4}$ F. Tang, D.-L. Liu, D.-X. Ye, Y.-P. Zhao, T.-M. Lu, G.-C. Wang, and A. Vijayaraghavan, J. Appl. Phys. 93, 4194 (2003).

${ }^{5}$ F. Tang, D.-L. Liu, D.-X. Ye, T.-M. Lu, and G.-C. Wang, J. Magn. Magn. Mater. 283, 65 (2004).

${ }^{6}$ A. Berger and M. R. Pufall, Appl. Phys. Lett. 71, 965 (1997).

${ }^{7}$ M. Schubert, T. E. Tiwald, and J. A. Woollam, Appl. Opt. 38, 177 (1999).

${ }^{8}$ D. Schmidt, B. Booso, T. Hofmann, E. Schubert, A. Sarangan, and M. Schubert, Appl. Phys. Lett. 94, 011914 (2009).

${ }^{9}$ D. Schmidt, B. Booso, T. Hofmann, E. Schubert, A. Sarangan, and M. Schubert, Opt. Lett. 34, 992 (2009).

${ }^{10}$ Handbook of Ellipsometry, edited by H. G. Tompkins and E. A. Irene (Springer, Heidelberg, 2004).

${ }^{11}$ M. Dressel, B. Gompf, D. Faltermeier, A. K. Tripathi, J. Pflaum, and M. Schubert, Opt. Express 16, 19770 (2008).

${ }^{12} \mathrm{M}$. Schubert, Infrared Ellipsometry on Semiconductor Layer Structures (Springer, Heidelberg, 2004).

${ }^{13}$ Š. Višňovský, Czech. J. Phys., Sect. B 36, 625 (1986).

${ }^{14}$ W. A. McGahan, P. He, J. A. Woollam, and F. O. Sequeda, Appl. Phys. Commun. 11, 375 (1992)

${ }^{15}$ R. M. Osgood, K. T. Riggs, A. E. Johnson, J. E. Mattson, C. H. Sowers, and S. D. Bader, Phys. Rev. B 56, 2627 (1997).

${ }^{16}$ D. Schmidt, E. Schubert, and M. Schubert, a detailed presentation of the MDF procedure and parameters will be published elsewhere.

${ }^{17}$ D. Weller, G. R. Harp, R. F. C. Farrow, A. Cebollada, and J. Sticht, Phys. Rev. Lett. 72, 2097 (1994).

${ }^{18}$ A. K. Zvezdin and V. A. Kotov, Modern Magnetooptics and Magnetooptical Materials (IOP, Bristol, 1997). 